\title{
Pseudonondiffracting slitlike beam and its analogy to the pseudonondispersing pulse
}

\author{
Joseph Rosen, Boaz Salik, and Amnon Yariv \\ California Institute of Technology, M/S 128-95, Pasadena, California 91125 \\ Hua-Kuang Liu \\ Jet Propulsion Laboratory, M/S 303-310, California Institute of Technology, Pasadena, California 91109
}

Received September 27, 1994

\begin{abstract}
A new nonspreading beam is proposed for the case in which diffraction occurs only in one transverse coordinate. The beam has the shape of a pulse in one dimension and is constant in the other (slitlike shape). The intensity of the pulse's peak remains almost constant along a finite interval on the propagation axis. The proposed beam is analyzed and demonstrated experimentally. The analogy between this beam and the temporal pulse in a dispersive medium is discussed.
\end{abstract}

The analogy between temporal pulse propagation in a dispersive medium and spatial Fresnel diffraction has recently yielded new methods to process short pulses. $^{1-4}$ One diffraction phenomenon that has not been imitated in the time domain is the so-called nondiffracting beam. ${ }^{5}$ The original nondiffracting beam, also called the Bessel beam, is a solution, of the form $E(r, z)=\exp (j \beta z) J_{0}(\alpha r)$, to the free-space scalar wave equation in which $\alpha^{2}+\beta^{2}=k^{2}, k$ is the wave number, $J_{0}$ is the zero-order Bessel function, and $(r, \theta, z)$ are the cylindrical coordinates. This solution is attractive because the intensity distribution in the entire space is independent of the $z$ coordinate and the transverse intensity profile has the shape of a pulse (beamlike shape). This solution, however, exists in three-dimensional space, whereas the temporal pulse lives in the two-dimensional (2D) time propagation axis domain $(t, z)$. Therefore, the analogy of the temporal dispersive domain holds completely only for the 2-D spatial diffractive domain $(x, z)$. As we see, reducing the dimensions of the Bessel beam strictly from $(r, \theta, z)$ to $(x, z)$ does not lead to satisfactory results. The aim of this Letter is to point out a new kind of pseudonondiffracting (PND) beam, characterized by a constant intensity along the propagation axis $z$ and a beamlike shape in one of its transverse dimensions, say, $x$. Based on the space-time analogy, and having a PND slitlike beam, we can announce the discovery of a novel pseudonondispersing temporal pulse, whose peak value remains constant along some predetermined propagation distance. The following treatment refers to the spatial case, and the temporal pulse will be considered after that.

Using Fresnel's approximation, we calculate the 2-D complex amplitude distribution around focal plane $\mathrm{P}_{f}$ (Fig. 1) to be

$$
\begin{aligned}
u(x, z)= & \frac{\exp [j k(z+2 f)]}{\sqrt{j \lambda f}} \\
& \times \int_{-\infty}^{\infty} g\left(x_{i}\right) \exp \left[-j \frac{k}{f}\left(\frac{z x_{i}{ }^{2}}{2 f}+x x_{i}\right)\right] \mathrm{d} x_{i},
\end{aligned}
$$

where $k=2 \pi / \lambda, \lambda$ is the wavelength, $f$ is the focal length of the cylindrical lens, $x_{i}$ is the transverse coordinate of plane $\mathrm{P}_{i}$, and $(x, z)$ are the coordinates behind the lens, with the focus as their origin. The longitudinal profile is obtained by substituting $x=0$ into Eq. (1):

$$
\begin{aligned}
u(0, z)= & \frac{\exp [j k(z+2 f)]}{\sqrt{j \lambda f}} \\
& \times \int_{-\infty}^{\infty} g\left(x_{i}\right) \exp \left(-j \frac{k z x_{i}^{2}}{2 f^{2}}\right) \mathrm{d} x_{i} .
\end{aligned}
$$

We look for a field distribution $u(x, z)$ with the shape of a pulse, i.e., $|u(0, z)|^{2}>>|u(x, z)|^{2}$, for all $x>W_{p}$, where $W_{p}$ is the width of the pulse and the axial distribution of the intensity is constant along a finite interval $\Delta z$, i.e., $|u(x=0, z)|^{2}=$ const. for $z \in \Delta z$. A function with useful properties for our purposes is of the form

$$
g\left(x_{i}\right)=\exp \left\{-j 2 \pi\left[\left|\frac{x_{i}}{b}\right|^{p}-\left(\frac{x_{i}}{a}\right)^{2}\right]\right\}, \quad p>2,
$$

where $a, b$, and $p$ are real positive parameters of the beam. When Eq. (3) is substituted into Eq. (2), the method of stationary phase approximation ${ }^{7}$ yields

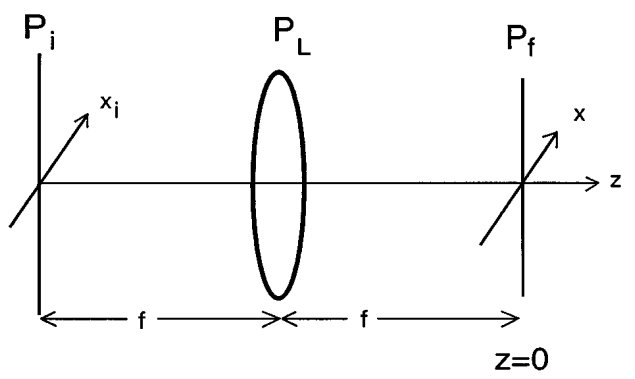

Fig. 1. Schematic system used to obtain the PND beam. 


$$
u(0, z) \approx \begin{cases}\frac{B \exp (j k z)}{\sqrt{2\left(\zeta-1 / a^{2}\right)}} & \zeta<-\alpha \Lambda+\frac{1}{a^{2}} \\ \exp (j k z)\left[C+D\left(\zeta-\frac{1}{a^{2}}\right)\right] & -\alpha \Lambda+\frac{1}{a^{2}}<\zeta<\Lambda+\frac{1}{a^{2}} \\ \frac{B \exp (j k z)}{\sqrt{2\left(\zeta-1 / a^{2}\right)}}\left(\frac{1}{\sqrt{2}} \exp \left\{-j 2 \pi\left[\left(\zeta-\frac{1}{a^{2}}\right)\left(\frac{2 b^{2}}{p}\right)\right]^{p /(p-2)}(1+p)\right\}+1\right) & \Lambda+\frac{1}{a^{2}}<\zeta\end{cases}
$$

where $\zeta=z / 2 \lambda f^{2} ; \quad \Lambda=0.5 b^{-2} p[4(1+p)]^{(2-p) / p}$; $B, C, D$ are complex constants whose precise value is not important here; and $0<\alpha<1$. Note that the functional behavior of $u(0, z)$ is different in each of the three regions. At the central region $u(0, z)$ is approximately linear. The length of this region is $\Delta z=2(1+\alpha) \Lambda \lambda f^{2}$, and its center is located at $\lambda f^{2}\left[\Lambda(1-\alpha)-2 a^{-2}\right]$ from the focal point. In the same manner it can be shown that substituting the conjugate function of Eq. (3) into Eq. (2) yields the opposite profile, $u^{*}(0,-z)$, of that given in relation (4). Therefore, by taking a mask of the combination $g\left(x_{i}\right)+g^{*}\left(x_{i}\right)$, where $a=\sqrt{2}[(1-\alpha) \Lambda]^{-1 / 2}$, we can combine the two linear curves with opposite slope signs to create a constant distribution. The length of the constant-intensity region is equal to that of the linear region.

The transverse shape of the beam near the focus is our next consideration. For mathematical convenience we investigate the beam at the point $z_{0}=0$. Substituting $z_{0}$ and $g\left(x_{i}\right)+g^{*}\left(x_{i}\right)$ [from Eq. (3)] into Eq. (1) yields

$$
\begin{aligned}
u\left(x, z_{0}\right)= & \frac{2 \exp \left[j k\left(z_{0}+2 f\right)\right]}{\sqrt{j \lambda f}} \\
& \times \int_{-\infty}^{\infty} \cos \left\{2 \pi\left[\left(\frac{x_{i}}{b}\right)^{p}-\left(\frac{x_{i}}{a}\right)^{2}\right]\right\} \\
& \times \exp \left(-j \frac{k}{f} x x_{i}\right) \mathrm{d} x_{i} .
\end{aligned}
$$

Assuming an infinite aperture, this integral can be approximated $^{7}$ at the region far from the origin $(x>>0)$ as

$$
u\left(x, z_{0}\right) \propto x^{2-p / 2(p-1)} \cos \left[2 \pi(1-p)\left(\frac{b x}{p \lambda f}\right)^{p /(p-1)}\right] .
$$

For an infinite aperture the envelope of the amplitude of the pulse decreases as $x^{(2-p) / 2(p-1)}$. A cumbersome analysis shows that this asymptotic behavior (for $x>>0$ ) stays valid for any $z \in \Delta z$, although the field distribution changes substantially. When the finite aperture $D$ is taken into account, one can estimate the overall width of the beam at $z_{0}$ by calculating the maximum spatial frequency of $g\left(x_{i}\right)$ at the point $x_{i}=D / 2$, and that is $W_{0}=\lambda f p D^{p-1} / 2^{p-1} b^{p}$. On the other hand, the width of the pulse (the sharp central lobe of the beam) is approximated under the assumption that its shape around the origin is determined mainly by the Fourier transform of the central lobe of $g\left(x_{i}\right)$. Therefore, the width of the pulse $W_{p}$ (at the focus) is $W_{p}=2 \lambda f / H$, where $H$ is the width of the central lobe of $g\left(x_{i}\right)$ obtained as the solution of the equation $(H / 2 b)^{p}-(H / 2 a)^{2}=1 / 4$. Note that only the overall width of the beam $W_{0}$ is related to the aperture width $D$. The shape of the pulse itself $\left(W_{p}, \Delta z\right)$ is independent of the aperture width if $D$ is above some value that empirically is found to be twice the width of the first cycle of $g\left(x_{i}\right)$. A complete simulation of the PND beam was conducted for $p=4, b=128$ pixels, $a=250$ pixels, and $\left(2 \lambda f^{2}\right)=128^{3}$ pixel $^{3}$. The behavior of the wave along the propagation axis can be studied from the solid curve of Fig. 2. For comparison, the longitudinal intensity of an ordinary focused beam resulting from the central lobe of $g\left(x_{i}\right)$ only [i.e., $\operatorname{rect}\left(x_{i} / H\right) g\left(x_{i}\right)$ ] is shown by the dashed curve. The latter beam is chosen for comparison with the PND beam because the two have similar transverse shapes around the focal point. The insets of Fig. 2 show the transverse profiles of the PND beam (solid curves) and the ordinarily focused beam (dashed curves). Although their width $W_{p}$ is equal, the PND beam keeps its beamlike shape for a longer distance than the ordinary focused beam.

Next, we demonstrate the PND beam experimentally. Looking at Fig. 1, we note that if $g\left(x_{i}\right)$ is illuminated by a plane wave, the field distribution at plane $\mathrm{P}_{L}$ just behind the lens is

$$
\begin{aligned}
& t\left(x_{L}\right)=\left[g^{*} L(f)\right] L(-f), \\
& L(f)=\exp \left(j \frac{k x^{2}}{f}\right) .
\end{aligned}
$$

Therefore the combination of the mask $g\left(x_{i}\right)$ plus the
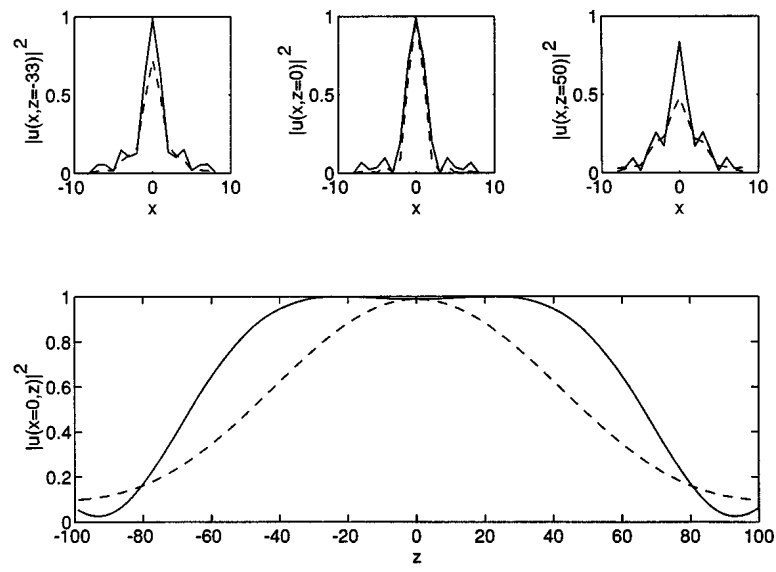

Fig. 2. Computer-simulated axial intensity distribution of the PND beam (solid curve) and the ordinary focused beam (dashed curve). The insets show the transverse cross section of the PND beam (solid curves) and the ordinary focused beam (dashed curves) at some points along the axis. 
(a)
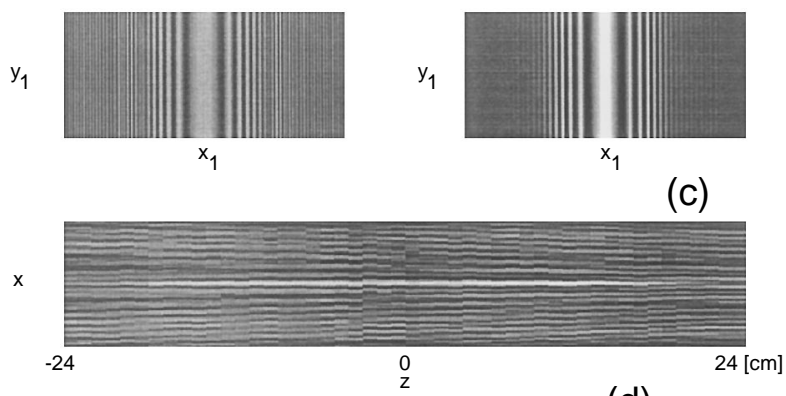

(d)

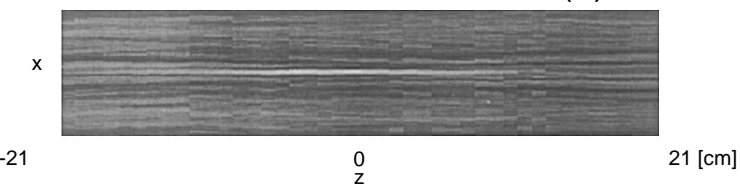

Fig. 3. Holograms that produce (a) the PND beam and (b) the ordinary focused beam. The 2-D intensity distribution of (c) the PND and (d) the ordinary focused beam.

lens can be replaced by a single mask with the distribution $t\left(x_{L}\right)$. For an amplitude-only transparency the mask that we use is given by

$$
s\left(x_{L}\right)=1+\frac{1}{2} t\left(x_{L}\right)+\frac{1}{2} t^{*}\left(x_{L}\right) .
$$

Assuming that $t\left(x_{L}\right)$ is normalized, $s\left(x_{L}\right)$ is a real positive distribution that is easily implemented. We see from Eq. (8), however, that there are two useless terms, $t^{*}\left(x_{L}\right)$ and a constant term. Fortunately, $t^{*}\left(x_{L}\right)$ is responsible for the appearance of a virtual image on plane $\mathrm{P}_{i}$, so it contributes only a weak scattering light around plane $\mathrm{P}_{f}$. A similar but more uniform contribution is due to the constant term.

The hologram used in the experiment is shown in Fig. 3(a). We obtained the distribution of the hologram by substituting $g\left(x_{i}\right)=\cos \left\{2 \pi\left[\left(x_{i} / b\right)^{4}-\right.\right.$ $\left.\left.\left(x_{i} / a\right)^{2}\right]\right\}\left(x_{i}<D / 2\right)$ into Eqs. (7) and (8), where $\lambda=$ $0.63 \mu \mathrm{m}, b=5 \mathrm{~mm}, a=9.77 \mathrm{~mm}, D=16 \mathrm{~mm}$, and $f=1.2 \mathrm{~m}$. The behavior of the PND beam passing the hologram around the distance $1.2 \mathrm{~m}$ is demonstrated in Fig. 3(c). As was done in the simulation, the performance of the PND beam is compared with the ordinary focused beam shown in Fig. 3(d). We created this beam by illuminating the mask shown in Fig. 3(b) by a plane wave. We obtained the mask of Fig. 3(b) by the same projection process as for the mask of Fig. 3(a), but this time the initial distribution was the central lobe only of $g\left(x_{i}\right)$.

The analogy between temporal pulse propagation in a dispersive medium and Fresnel diffraction is our next consideration. Based on Ref. 3, the pulse is represented as a sum of plane waves, each propagating with a different phase delay $\beta(\omega)$, and $\beta(\omega)$ is approximated as a polonium series, $\beta(\omega)=\beta_{0}+$ $\beta_{1} \omega+\beta_{2} \omega^{2} / 2$, where $\omega$ is the angular frequency. With these assumptions the field of the pulse is

$$
\begin{aligned}
u(t, z)= & \exp \left(j \beta_{0} z\right) \int_{-\infty}^{\infty} U(\omega) \exp \left(-j \frac{\beta_{2} z}{2} \omega^{2}\right) \\
& \times \exp \left[j \omega\left(t-\beta_{1} z\right)\right] \mathrm{d} \omega .
\end{aligned}
$$

The pulse distribution is represented as a Fourier transform of its temporal spectrum (at $z=0$ ) multiplied by a quadratic phase factor. According to the convolution theorem, it can be represented by a convolution integral,

$$
u(t, z)=\frac{\exp \left(j \beta_{0} z\right)}{\sqrt{2 j \pi \beta_{2} z}} \int_{-\infty}^{\infty} u\left(t^{\prime}\right) \exp \left[j \frac{\left(t-t^{\prime}\right)^{2}}{2 \beta_{2} z}\right] \mathrm{d} t^{\prime}
$$

where $t=t-\beta_{1} z$, i.e., the time variable minus the delay that the pulse takes to reach to $z$. This expression has the same form of the Fresnel integral describing the propagation of an electromagnetic field in free space, where $t$ is equivalent to $x$. Based on this analogy, there must be an equivalent expression to Eq. (1) in the $(t, z)$ domain. To obtain it, assume that the initial pulse distribution exists at a distance $f$ from a temporal lens of the form $\exp \left(-j t^{2} / 2 \beta_{2} f\right)$. After the analyses of Ref. 6 , the pulse distribution after it propagates the distance $f$ and is multiplied by the temporal lens (at time $t=\beta_{1} f$ ) is

$$
\begin{aligned}
u(t, z)= & \frac{\exp \left[j \beta_{0}(z+2 f)\right]}{\sqrt{2 j \pi \beta_{2} f}} \\
& \times \int_{-\infty}^{\infty} u\left(t^{\prime}\right) \exp \left[-\frac{j}{\beta_{2} f}\left(\frac{t^{\prime} z}{2 f}-t t^{\prime}\right)\right] \mathrm{d} t^{\prime},
\end{aligned}
$$

where the temporal lens is at $z=-f$. Equation (11) has indeed the same form as Eq. (1). Therefore, in general we can reshape a profile of the pulse along the propagation axis, inside a dispersive medium, in the same manner as has been done to the beam in the spatial domain. ${ }^{6,8}$ Specifically, we can create a pseudonondispersive temporal pulse by using the theory of the pseudonondiffracting beam in the 2-D space analyzed at the beginning of this Letter. Analogously to the spatial pulse, we expect the temporal pulse to remain constant for a distance $\Delta z=4 \pi \Lambda(1+$ a) $\beta_{2} f^{2}$ if a pulse of the form $\cos \left\{2 \pi\left[(t / b)^{p}-(t / a)^{2}\right]\right\}$ is processed by the temporal analogy of Eqs. (7) and (8).

This research was sponsored in part by the NASA Office of Advanced Concepts and Technology.

\section{References}

1. E. B. Treacy, IEEE J. Quantum Electron. QE-5, 454 (1969).

2. B. H. Kolner and M. Nazarathy, Opt. Lett. 14, 630 (1989).

3. A. W. Lohmann and D. Mendlovic, Appl. Opt. 31, 6212 (1992).

4. M. T. Kauffman, W. C. Banyai, A. A. Godil, and D. M. Bloom, Appl. Phys. Lett. 64, 270 (1994).

5. J. Durnin, J. J. Miceli, Jr., and J. H. Eberly, Phys. Rev. Lett. 58, 1499 (1987).

6. J. Rosen and A. Yariv, Opt. Lett. 19, 843 (1994).

7. A. Papoulis, Systems and Transforms with Applications in Optics, 1st ed. (McGraw-Hill, New York, 1968), Chap. 7, p. 222.

8. J. Rosen, Opt. Lett. 19, 369 (1994).

9. R. Piestun and J. Shamir, Opt. Lett. 19, 771 (1994). 